\title{
RELATIONSHIP OF THE ${ }^{3} P_{0}$ DECAY MODEL TO OTHER STRONG DECAY MODELS
}

\author{
B. DESPLANQUES, A. NICOLET AND L. THEUSSL \\ Institut des Sciences Nucléaires (UMR CNRS/IN2P3-UJF), \\ F-38026 Grenoble Cedex, France \\ E-mail: desplanq@isn.in2p3.fr
}

\begin{abstract}
The ${ }^{3} P_{0}$ decay model is briefly reviewed. Possible improvements, partly motivated by the examination of a microscopic description of a quark-anti-quark pair creation, are considered. They can provide support for the one-body character of the model which, otherwise, is difficult to justify. To some extent, they point to a boost effect that most descriptions of processes involving a pair creation cannot account for.
\end{abstract}

\section{Introduction}

The ${ }^{3} P_{0}$ decay model, first introduced by Micul, has been subsequently applied to the description of many processes by Le Yaouanc et al.2. Since then, it has been used extensively with a reasonable success, especially for the hadronic decays of mesons. Being described by a one-body operator, the model can be employed easily, while its strength is generally fitted to experiment. In these conditions, the agreement is not much better than a factor 2, which is too large to make stringent tests of the description of hadrons for instance. Improvements should therefore be introduced. This however requires to understand what the model accounts for. After reviewing the model, we will consider possible improvements, based on a microscopic description of a quark-anti-quark pair creation.

\section{The ${ }^{3} P_{0}$ decay model}

The ${ }^{3} P_{0}$ decay model assumes a creation of a quark-anti-quark pair from the vacuum with the corresponding quantum numbers, $J=0, L=1, S=1$, $T=0$. Represented in Fig. 17a for a meson decay, it may be described by the following operator:

$$
H_{\text {pair }}=\gamma \sum_{i, j} a_{i}^{\dagger}(\vec{p}) b_{j}^{\dagger}\left(\vec{p}^{\prime}\right) \frac{\vec{\sigma} \cdot\left(\vec{p}-\vec{p}^{\prime}\right)}{2 \sqrt{2 \pi}}(2 \pi)^{3} \delta\left(\vec{p}+\vec{p}^{\prime}\right)+h . c .
$$

In comparison with the elementary emission model shown in Fig. 17b, where a meson is emitted from a quark line, it offers the considerable conceptual 


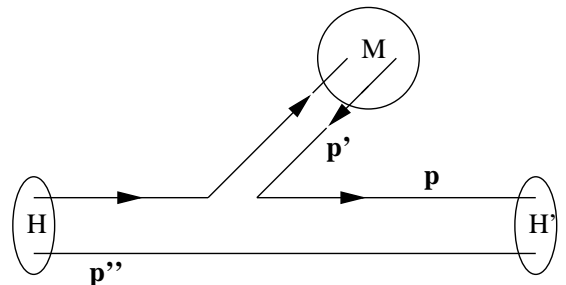

a

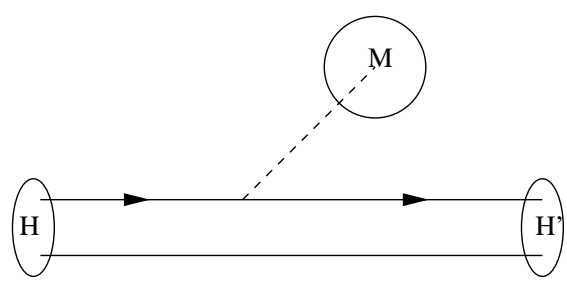

b

Figure 1. Diagrams representing a meson decay with a one-body interaction: within the ${ }^{3} P_{0}$ decay model (a) and an elementary emission model (b).

advantage that all hadrons are considered on the same footing.

Among improvements, it has been proposed 3 to introduce some momentum dependence in the strength $\gamma$. A relativized version of the model has also been considered, involving the replacement of $\vec{\sigma} \cdot\left(\vec{p}-\vec{p}^{\prime}\right)$ in Eq. (1) by $2 m \bar{u}(\vec{p}) v\left(\vec{p}^{\prime}\right)$. Some improvement is obtained but in absence of insight on the origin of the model, one cannot draw firm conclusions.

Although it should be used in the c.m. of the decaying system, the creation of a pair from the vacuum without relation to the other particles is difficult to imagine. Moreover, a term like Eq. (11) can be absorbed in a redefinition of the quarks and their masses, producing elementary quark-meson couplings for instance. Kokosky and Isgur discussed the possibility that the ${ }^{3} P_{0}$ decay model could account for a flux-tube breaking in some limit 5 . The strength is not known however. Another possibility js that the pair creation is closely related to the interaction between quarks 6 . This hypothesis, that we will develop in the following, is illustrated in Fig. 2a for a meson decay.

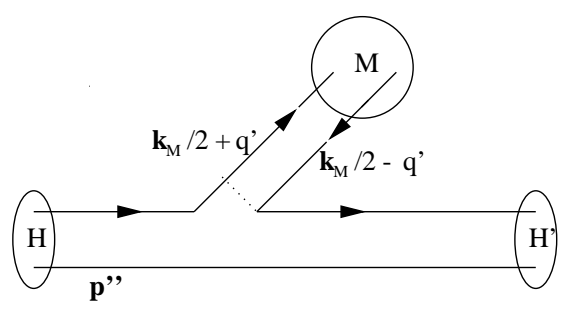

a

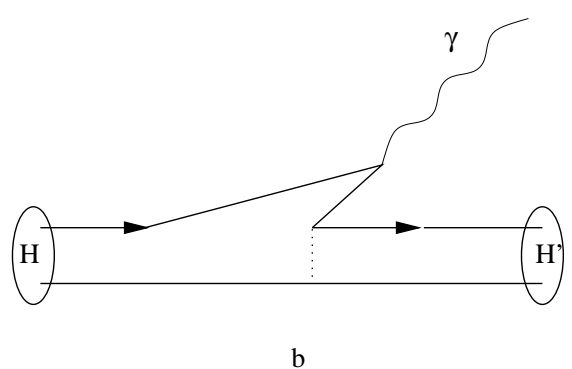

Figure 2. Diagrams representing a meson decay with a two-body interaction: emission of a meson (a) and a photon (b). 


\section{$3 \quad$ A key relationship}

The calculation of the decay amplitude of a hadron $H$ into another one $H^{\prime}$ and a meson $M$, as depicted in Fig. 2a for a particular case, implies an expression like the following:

$$
A_{\left(H \rightarrow H^{\prime}+M\right)} \propto \int \frac{d \vec{p}^{\prime \prime}}{(2 \pi)^{3}} \psi_{H}^{*}(\ldots) \psi_{H^{\prime}}(\ldots) \int \frac{d \vec{q}^{\prime}}{(2 \pi)^{3}} V\left(\vec{q}, \vec{q}^{\prime}\right) \phi_{M}\left(\vec{q}^{\prime}\right),
$$

where $\phi_{M}(\vec{q})$ represents the meson wave function. $V\left(\vec{q}, \vec{q}^{\prime}\right)$ represents the interaction responsible for a pair creation. Quite generally, its expression is complicated and without relation to the interaction between quarks. In one case, however, such a relation can be established. For spin-less particles, both interactions are the same (non-relativistic approximation). One can then use the equation that the meson wave function has to fulfill:

$$
\int \frac{d \vec{q}^{\prime}}{(2 \pi)^{3}} V\left(\vec{q}, \vec{q}^{\prime}\right) \phi_{M}\left(\vec{q}^{\prime}\right)=\left(E-2 e_{q}\right) \phi_{M}(\vec{q}) .
$$

This can be used to replace the last factor in Eq. (22) by the wave function itself, obtaining:

$$
A_{\left(H \rightarrow H^{\prime}+A\right)} \propto \int \frac{d \vec{p}^{\prime \prime}}{(2 \pi)^{3}} \psi_{H}^{*}(\ldots) \psi_{H^{\prime}}(\ldots) "\left(E-2 e_{q}\right) " \phi_{A}(\vec{q}),
$$

where $\vec{q}$ has to be replaced appropriately in terms of the external momenta and $\vec{p}^{\prime \prime}$. This last expression looks very much like the one used when employing the ${ }^{3} P_{0}$ decay model (spin put apart). It does not contain the interaction explicitly but involves a one-body operator. This one however appears with a well defined factor, " $E-2 e_{q}$ ", providing a clue for both the strength and the momentum dependence, often introduced on a phenomenological basis.

The expression of the above amplitude corresponds to a particle-antiparticle pair creation that has the same form as the one appearing in a freeparticle interaction. It however differs by essential features. As already mentioned, such a term can be absorbed into the redefinition of the particle fields. The functional dependence of the front factor, " $E-2 e_{q}$ ", rules out this transformation here, since it cancels for free particles, consistently with the fact that it originates from an interaction term. This can solve one of the problems with the ${ }^{3} P_{0}$ decay model, namely the creation of a pair without relation to the environment.

With the above respect, it is worthwhile to mention another example where an interaction effect can be turned into a single-particle contribution. It concerns the Z-type contribution to the emission of a photon shown in Fig. 2 $\mathrm{b}$. This one evidently involves the interaction. However when the full 
Feynman diagram corresponding to this figure is considered, it is found that the same contribution appears as a single-particle one. Consistency is achieved by the fact that this contribution involves a factor similar to the abgve one, " $E-2 e_{q}$ ". The details can be checked by looking at a simple modelt.

When applying the above ideas to spin $1 / 2$ particles, one has to take into account that many terms of the order $\vec{\sigma} \cdot \vec{p}$ are produced by the interaction, besides those appearing at the vertex where a quark-anti-quark pair is created. While the latter ones can be accounted for by the ${ }^{3} P_{0}$ decay model, the other ones cannot. For that particular contribution, which could represent some average one, the structure of the operator is obtained as above for spin-less particles and is given by the replacement in Eq. (11):

$$
\gamma \frac{\vec{\sigma} \cdot\left(\vec{p}-\vec{p}^{\prime}\right)}{2 \sqrt{2 \pi}} \rightarrow "\left(E-2 e_{q}\right) " \bar{u}(\vec{p}) v\left(\vec{p}^{\prime}\right) .
$$

If the meson is strongly bound ( $E$ negligible) and $e_{q}$ approximated by the quark mass, the value $2 \sqrt{2 \pi} \simeq 5$ is obtained for $\gamma$. This value compares well to the value that can be obtained from meson necays 6 but is significantly smaller than the one derived from baryon decayst 6 . Within conventions, the sign that is required in some cases is also known.

A relativistic approach based on the description of mesons by BetheSalpeter amplitudes suggested some similarity with the quark-pair creation

model 1 . Comparison with the present work indicates a close relation which strongly supports the hypothesis that the ${ }^{3} P_{0}$ decay model, as completed here, would implement boost effects that an approximate (non-relativistic) description of hadrons cannot account for. The possibility that it still accounts for other effects remains open however.

\section{References}

1. L. Micu, Nucl. Phys. B 10, 521 (1969).

2. A. Le Yaouanc et al., Hadron Transitions in the Quark Model (Gordon and Breach, 1988).

3. W. Roberts and B. Silvestre-Brac, Phys. Rev. D 57, 1694 (1998).

4. F. Cano et al., Z. Phys. A 359, 315 (1997).

5. R. Kokoski and N. Isgur, Phys. Rev. D 35, 907 (1987).

6. E.S. Ackleh, T. Barnes and E.S. Swanson, Phys. Rev. D 54, 6811 (1996).

7. B. Desplanques, L. Theußl and S. Noguera, Phys. Rev. C 65, 038202 (2002).

8. L. Theußl et al., Eur. Phys. J. A 12, 91 (2001).

9. D.S. Kulshreshtha and A.N. Mitra, Phys. Rev. D 28, 588 (1983). 